\title{
Modelos de mercadotecnia como una herramienta para las Pymes en México
}

\section{Marketing models as a tool for SMES in Mexico}

\author{
Omaira Cecilia Martínez-Moreno* \\ Universidad Autónoma de Baja California - México \\ ORCID iD: https://orcid.org/0000-0002-9562-1840 \\ omairam@uabc.edu.mx
}

\section{Gabriela Mondragón-Hernández}

Universidad Autónoma de Baja California - México gmondragon@uabc.edu.mx

* Autor a quien debe ser dirigida la correspondencia
Fecha de recepción: 04/10/2019 Fecha de evaluación: 12/02/2020

Fecha de aceptación: 08/04/2020

Cómo citar: Martínez-Moreno, O., \& Mondragón-Hernández, G. (2020). Modelos de mercadotecnia como una herramienta para las Pymes en México. Revista Científica Anfibios, 3(1), 47-51. https://doi.org/10.37979/afb.2020v3n1.61

\section{Resumen}

En el presente artículo se realiza un análisis de los modelos básicos de mercadotecnia como una herramienta para el desarrollo de un plan de mercadotecnia para las Pymes en México, considerando los cuatro conceptos básicos de Producto, Precio, Plaza y Promoción, y los retos que representa para su aplicación desde el nacimiento y desarrollo de este modelo de empresas, las cuales, bien se sabe que tienen una alta tasa de desaparición en México, y sin dejar de lado que los empresarios pueden encontrar en la mezcla de mercadotecnia una más rápida colocación de sus productos o servicios, y lo que buscan toda empresa que es la rentabilidad del negocio y la permanencia en el mercado.

\section{Palabras claves:}

Mercadotecnia; Producto; Precio; Plaza; Promoción

\begin{abstract}
This article analyses the basic marketing models as a tool for the development of a marketing plan for SMEs in Mexico, considering the four basic concepts of Product, Price, Place and Promotion, and the challenges it represents for its application since the birth and development of this business model, which are well known to have a high rate of disappearance in Mexico, and without forgetting that entrepreneurs can find in the marketing mix a faster placement of their products or services, and what every company is looking for which is the profitability of the business and permanence in the market.
\end{abstract}

\section{Keywords:}

Marketing; Product; Price; Place; Promotion 


\section{Introducción}

En la actualidad no hay un mercado donde no exista competencia, donde las empresas esperen que los clientes lleguen a querer comprar los productos; en un mundo tan globalizado, con tanta información disponible inmediata para todos, los gerentes de empresas tienen el reto de distinguirse del resto de las demás compañías, es por eso que se ha recurrido a la Mercadotecnia dirigida tanto: a los clientes internos con la finalidad de generar sentido de pertenencia para con la empresa (Arbelaez, Castillo \& Licona, 2017), como hacia los clientes externos o finales con el propósito de lograr tomar una posición y un prestigio como marca de un producto o servicio.

Hace algunos años el enfoque era totalmente hacia las características y bondades del producto; pero ahora se va más allá, se busca la total satisfacción del cliente, con el interés de ayudarle a tomar la mejor decisión de compra que lo lleve a generar una fidelidad a la marca, sin importar si es un producto tangible o un servicio; las empresas están buscando cada vez más conocer más acerca de las necesidades de sus clientes para poderles resolver.

\section{La Mercadotecnia}

Para entender de dónde vienen todos estos cambios en que han tenido que atender los nuevos gerentes en las empresas de hoy en día, habría que recurrir a la definición de Mercadotecnia, la cual según La American Marketing Association ofrece la siguiente definición formal: Marketing es la actividad o grupo de entidades y procedimientos para crear, comunicar, entregar e intercambiar ofertas que tienen valor para los consumidores, clientes, socios y la sociedad en general (Kotler, 2012)

Los especialistas en marketing deben decidir qué características deben incluirse en el diseño de un nuevo producto o servicio, los precios con los que se va a comercializar, dónde vender los productos $\mathrm{u}$ ofrecer los servicios y cuánto se debe de invertir en publicidad, ventas, Internet; y además las decisiones que se tomen ahora son dentro de entorno de constante cambio, en el cual los consumidores, su competencia y desde luego el internet y la tecnología juegan un papel preponderante, esto evidentemente a través de las redes sociales, donde la respuesta de una empresa puede ser volátil y multiplicarse rápidamente con sus clientes y público en general.

Para comprender de una mejor manera la función de la Mercadotecnia, hay que entender los conceptos fundamentales del Marketing que son Necesidades, Deseos y Demandas.

Los seres humanos tenemos necesidades básicas para nuestra subsistencia como son el aire, alimento, agua, vestido y refugio; además de las necesidades de ocio, recreación y entretenimiento, las cuales se convierten en deseo una vez que se dirigen a un objeto físico que puede satisfacer la necesidad, lo que nos lleva a una definición muy corta y sencilla del Marketing; "satisfacer las necesidades de manera rentable". (Kotler, 2012)

\section{Tipos de modelos de mercadotecnia}

En los años ochenta, la mercadotecnia se basaba en la aplicación de las 4Ps, también conocidas como el mix de mercadotecnia (marketing mix). Estos cuatro conceptos eran considerados estratégicos al momento de decidir lanzar o reposicionar un producto / servicio en el mercado y en la mente del consumidor. Era fundamental la correcta aplicación de estos elementos para que la estrategia funcionara y llegar de manera efectiva a lograr alguna reacción en el mercado meta.

Los elementos de la mezcla de marketing propuesta por McCarthy, Shapiro y Perreault (1979) citado por Gordon (2012) fueron:

Producto: es un objeto tangible o servicio intangible que se produce o fabrica y se ofrece a los consumidores en el mercado.

Precio: es la cantidad que un consumidor paga por el producto o servicio, normalmente un costo económico.

Lugar: representa la ubicación donde se puede comprar un producto o servicio y, a menu- 
do, se puede denominar como el canal de distribución. Esto puede incluir tiendas físicas y puntos de venta virtuales en línea.

Promoción: representa las comunicaciones que los profesionales de marketing utilizan en el mercado, incluida la publicidad, las relaciones públicas, la venta personal y la promoción de ventas.

El objetivo de los empresarios en las operaciones de marketing es encontrar una oferta de productos y servicios que incremente el nivel de satisfacción del comprador y genere utilidades para la empresa en este Mix de Mercadotecnia se agregó el Servicio como un quinto elemento por considerar que el paquete de valor está compuesto por los beneficios en los que el servicio es un componente de valor agregado que puede constituir una ventaja competitiva. (Gordon, 2012)

A partir del Modelo propuesto de las 4P's se analizó el comportamiento del consumidor y del cliente para la toma de decisión de compra y como resultado se agregaron otros tres elementos adicionales muy importantes "People, Process y Physical Evidence" (Marchione, 2012)

\section{De los cuales se explica a continuación:}

People: (Personas) este incluye todos los factores humanos involucrados en el modelo de negocios y no solo los empleados, si no también proveedores, directivos, gerentes, consumidores y clientes.

Procesos (Process): Este agrupa todo el encadenamiento de actividades que permiten que un producto o servicio llegue a un consumidor final y no solo al cliente.

Evidencia Física (Physical Evidence) se concentra en el ambiente y el entorno en el que un producto o servicio es entregado al cliente o consumido por el usuario final.

Con esta información se complementa en Modelo de las 7p's del Mix de Mercadotecnia, con el cual se deberán tomas las decisiones importantes sobre la estrategia del producto o servicio. Una interesante la aplicación de estos modelos de Mercadotecnia, la podemos visualizar en los emprendedores que a partir de una idea, deciden invertir para desarrollar una pequeña o media empresa, lo que se denomina en México como una Pyme.

Las Pymes en México constituyen el 97\% del total de las empresas, generadoras de empleo del $79 \%$ de la población y generan ingresos equivalentes al 23\% del Producto Interno Bruto (PIB), lo anterior es una clara señal de debemos poner atención a este tipo de empresas y verlas como lo que en realidad son: la base de la economía mexicana. (GEOGRAFÍA, 2015)

Para los emprendedores, el desarrollo y consolidación son los ejes fundamentales para el desarrollo del país. Estos utilizan el diseño, fomento y promoción de programas y herramientas para crear, consolidar y desarrollar a las pequeñas y medianas empresas que en suma representan el 99 por ciento de los negocios que existen en el país, y además generan cerca del 80 por ciento de los empleos. (Gobierno de México, 2006) Cuando se decide a abrir una empresa, lo último que se piensa es que como llegaras a los clientes, los emprendedores están tan ocupados en buscar recursos para hacer su negocio que lo último que piensan es en las oportunidades que tienen al conocer la Mercadotecnia para sus empresas; uno de los mayores retos de estos empresarios es contar con un presupuesto para implementar un buen plan de marketing; sin embargo esto no quiere decir que fracasen en su intento por hacer negocios.

Este tipo de empresas cuentan con grandes ventajas, pero las desconocen y aunado a esto deben de sobre salir del resto de las empresas pymes que salen día a día en el mercado compitiendo por los mismos clientes. Con el Marketing como herramienta, para las pymes sería mucho más fácil subsistir al índice de mortandad ya que de acuerdo a las estadísticas, se dice que de cada 100 empresas que nacen, 90 no llegan a los dos años de vida, lo cual es un dato interesante.

El panorama para las empresas pymes en México, es positivo y podría mejorar como bien se comentó anteriormente aplicando el 
modelo de las 7p's con un enfoque a desarrollar cada uno de los puntos que lleve al empresario a hacer conciencia que aun cuando la empresa este naciendo, desde su base se dé un enfoque a donde se desea llegar en un mediano y largo plazo.

Tabla 1. Retos de aplicación de las 4p's en una pyme mexicana

\begin{tabular}{cll}
\hline "P" & RETO & REALIDAD \\
\hline Producto & Conocer a fondo el producto o servicio & Los emprendedores están tan ocupados con bus- \\
& que se está ofreciendo, & y encontrar su ventaja competitiva del producto o \\
& & servicio que ofrecen, que los distinga del resto de \\
& la competencia en el mercado.
\end{tabular}

Precio El precio que está dispuesto a pagar el cliente

Lugar (Plaza) Ubicación donde se puede adquirir el producto o servicio

Promoción

Representa las comunicaciones que los profesionales de marketing utilizan en el mercado, incluida la publicidad, las relaciones públicas, la venta personal y la promoción de ventas.
Con las nuevas tecnologías, muchos emprendedores consideran que al tener información en las redes sociales $(\mathrm{FB})$ ya se venderán sus productos, situación que no siempre funciona para todos los giros.

Este concepto la mayoría de las ocasiones no se considera dentro del presupuesto de la empresa y genera gastos que no se controlan, como dar muestras, participar en eventos sin una estrategia de venta.

\section{Fuente: Elaboración propia}

Análisis de las 3P's adicionales del Modelo de Mezcla de Mercadotecnia

Considerando la aplicación de las siguientes variables P's importantes en la Mezcla de Mercadotecnia, para la empresa Pyme, podemos determinar el análisis siguiente:

People: (Personas) en este rubro la mayoría de las empresas pymes inician haciendo alianza con la misma familia, lo que en si representa un principal reto, y a la vez una oportunidad ya que, al ser una empresa pequeña, se puede tener contacto de inmediato con todos y cada uno de los involucrados como los empleados, proveedores, directivos, gerentes, consumidores y clientes.

Procesos (Process): el proceso de una empresa pequeña puede ser relativamente sencillo, identificando las actividades que hacen que la empresa funcione, para que llegue al usuario final reciba el producto o servicio.

Evidencia Física (Physical Evidence) este punto es importante para el seguimiento de la satisfacción total del cliente, ya que así se considera la manera en la que el producto o servicio es entregado al cliente o consumido por el usuario final.

\section{Conclusión}

Como parte de la planeación de una empresa Pyme, no es tarea sencilla considerar la realización de un plan de mercadotecnia, más aun cuando no se cuenta con un presupuesto y además no se tiene conocimiento de la importancia de dicha herramienta; lo que podría parecer algo imposible se puede resolver con un poco de atención por parte de los emprende- 
dores y nuevos empresarios, haciendo una pausa en el camino para analizar las bondades de su producto o servicio, así como las ventajas competitivas tanto de este como de su equipo de trabajo, para así segmentar adecuadamente al cliente que desea atender, conociendo sus necesidades y deseos, y lo haga distinguirse del resto de la competencia, logando con esto el acompañamiento y fidelidad de sus clientes, que lo lleven a ser recomendado de manera natural y espontanea reforzando su imagen en re- des sociales, y a la vez generar una comunidad que se identifique con sus productos o servicios, generando confianza y ofreciendo un reconocimiento como cliente distinguido, además de la fuerza que se puede obtener a través de las alianzas estratégicas que se pueden hacer con sus proveedores y cadena de suministros al trabajar en conjunto, para así fortalecer el crecimiento en un ambiente de sana competencia y permanencia en el mercado.

\section{Referencias}

Arbelaez, D. C., Castillo, E. M. E., \& Licona, J. M. (2017). Endomarketing. Revista Cultural Unilibre, (2), 50-57. https://doi.org/10.18041/1909-2288/revista_cultural.2.2017.4225

GEOGRAFÍA, I. (2015). Encuesta Nacional sobre Productividad y Competitividad de las Micro, Pequeñas y Medianas Empresas (ENAPROCE) 2015. http://www.inegi.org.mx/est/contenidos/proyectos/encuestas/establecimientos/otras/enaproce/default_t.aspx

Gobierno de México. (2006). Programa de Financiamiento a la Mediana Empresa Agroalimentaria y Rural (PROEM). https://www.gob.mx/fira/acciones-y-programas/programa-de-financiamiento-a-la-mediana-empresa-agroalimentaria-y-rural-proem

Gordon, R. (2012). Re-thinking and re-tooling the social marketing mix. Australasian Marketing Journal (AMJ), 20(2), 122-126. https://doi.org/10.1016/j.ausmj.2011.10.005

Kotler, P. Y. (2012). Dirección de Marketing. Pearson.

Marchione, J. C. (2012). ¿Las 7 P y las 5 C del Marketing empiezan por los costos o terminan con ellos?. Costos y gestión, (83), 21-34.

McCarthy, E. J., Shapiro, S. J., \& Perreault, W. D. (1979). Basic marketing (pp. 29-33). Ontario: Irwin-Dorsey. 